\title{
Astrocyte galectin-9 potentiates microglial TNF secretion
}

\author{
Andrew J Steelman and Jianrong Li ${ }^{*}$
}

\begin{abstract}
Background: Aberrant neuroinflammation is suspected to contribute to the pathogenesis of myriad neurological diseases. As such, determining the pathways that promote or inhibit glial activation is of interest. Activation of the surface glycoprotein T-cell immunoglobulin and mucin-domain containing protein 3 (Tim-3) by the lectin galectin-9 has been implicated in promoting innate immune cell activation by potentiating or synergizing toll-like receptor (TLR) signaling. In the present study we examined the role of the Tim-3/galectin-9 pathway in glial activation in vitro.

Method: Primary monocultures of microglia or astrocytes, co-cultures containing microglia and astrocytes, and mixed glial cultures consisting of microglia, astrocytes and oligodendrocytes were stimulated with poly(l:C) or LPS, and galectin-9 up-regulation was determined. The effect of endogenous galectin-9 production on microglial activation was examined using cultures from wild-type and Lgals9 null mice. The ability for recombinant galectin-9 to promote microglia activation was also assessed. Tim-3 expression on microglia and BV2 cells was examined by qPCR and flow cytometry and its necessity in transducing the galectin-9 signal was determined using a Tim-3 specific neutralizing antibody or recombinant soluble Tim-3.

Result: Astrocytes potentiated TNF production from microglia following TLR stimulation. Poly(l:C) stimulation increased galectin-9 expression in microglia and microglial-derived factors promoted galectin-9 up-regulation in astrocytes. Astrocyte-derived galectin-9 in turn enhanced microglial TNF production. Similarly, recombinant galectin-9 enhanced poly(l:C)-induced microglial TNF and IL-6 production. Inhibition of Tim-3 did not alter TNF production in mixed glial cultures stimulated with poly(l:C).

Conclusion: Galectin-9 functions as an astrocyte-microglia communication signal and promotes cytokine production from microglia in a Tim-3 independent manner. Activation of CNS galectin-9 likely modulates neuroinflammatory processes in which TNF and IL-6 contribute to either pathology or reparation.
\end{abstract}

Keywords: Galectin-9, TNFa, Astrocytes, Microglia activation, Poly(l:C), Neuroinflammation

\section{Background}

Reactive gliosis is associated with many neurodegenerative diseases and is considered a hallmark feature of neuroinflammatory diseases. It is well established that both microglia and astrocytes express various toll-like receptors (TLRs) [1], intracellular pathogen recognition receptors $[2,3]$, and danger signal receptors and are capable of responding to their respective ligands, in part through the production of chemokines, cytokines and growth factors $[4,5]$. Given that dendritic cells are absent from the brain

\footnotetext{
*Correspondence: jrli@cvm.tamu.edu

Department of Veterinary Integrative Biosciences and Institute for

Neuroscience, Texas A\&M University, Mail Stop 4458, College Station, TX 77843 , USA
}

parenchyma and that the central nervous system (CNS) is devoid of a draining lymphatic system, it is believed that following infection of the CNS the initial recognition of invading pathogens by resident glia is paramount in facilitating the attraction, stimulation, and regulation of both the innate and adaptive arms of the peripheral immune system within this organ [6,7]. Moreover, mounting evidence suggests that inflammation in the CNS, which is orchestrated by reactive gliosis, ushers the reparation of damaged tissues, much as occurs in the periphery $[8,9]$.

Because some products, in particular proinflammatory cytokines and reactive oxygen intermediates produced following glial activation, are potentially cytotoxic or result 
in intracellular stress, the magnitude, timing and duration of glial activation likely dictates the delicate balance between the beneficial and detrimental effects of neuroinflammatory responses. In humans, aberrant glial activation is associated with demyelinating diseases such as multiple sclerosis [10] as well as a broad spectrum of other neurological diseases including stroke [11], epilepsy [12-14], amyotrophic lateral sclerosis, Alzheimer's and Parkinson's diseases [15] and depression [16,17]. Likewise, in an animal model of multiple sclerosis, experimental autoimmune encephalomyelitis (EAE), microgliosis has been shown to contribute to disease onset and progression [18-20]. Furthermore, microglial production of the proinflammatory cytokines IL-6 and TNF appears to promote the development of seizures following experimental infection of the CNS with Theiler's murine encephalomyelitis virus [21-25]. Acute glial activation following either peripheral or central administration of low-dose lipopolysaccharide (LPS) has been shown to induce symptoms of depression in mice [26-28]. Therefore, uncovering the factors that regulate glial activation is pertinent to understanding CNS disease pathogenesis and the return to homeostasis. Importantly, while much research has focused on the ability of either microglia or astrocytes to respond to inflammatory stimuli, far fewer studies have investigated potential intercellular pathways that are exploited between glia to orchestrate inflammatory responses within the CNS.

Galectins are $\beta$-galactoside-binding proteins that exhibit a broad range of functions, in part through their ability to bind branched glycans on glycoconjugates causing lattice formation and clustering of lipid rafts [29-31]. Recently, galectins have gained increased recognition for their ability to influence immune responses, either by acting as pathogen recognition receptors, modulating innate immune responses or by regulating adaptive immunity [32]. However, the functional role of galectin expression, especially galectin-9, within the CNS is incompletely understood. Studies in vitro have shown that galectin-9 can induce maturation of human monocyte-derived dendritic cells [33] and enhance proinflammatory cytokine secretion [34], which have been attributable to its ability to bind the highly glycosylated T-cell immunoglobulin and mucin domain-containing protein 3 (Tim-3) [34]. While galectin-9 protein or the gene encoding galectin-9, $\operatorname{Lgals} 9$, is not normally expressed at high levels within the CNS [35,36], it is increased following experimental pneumococcal meningitis [37] as well as infection with herpes simplex virus [38] or Japanese encephalitis virus [39] and is reportedly increased in the brains of multiple sclerosis patients $[34,40]$, indicating that it becomes up-regulated during inflammatory conditions.

Herein, we report that astrocytes can promote microglial TNF production. Notably, recombinant galectin-9 potentiated both TNF and IL-6 secretion from microglia when concurrently administered with poly(I:C). Additionally, utilizing glia derived from $L$ gals $9^{-/}$mice we found that galectin-9 was, in part, sufficient to elicit this effect. Together, these results indicate that CNS-derived galectin-9 can function as an interglial signaling molecule and may promote neuroinflammatory processes by enhancing microglial TNF production.

\section{Methods and materials \\ Animals}

Rat glial cultures were obtained from Sprague Dawley rat pups (P1-2; Harlan; Houston, TX, USA). Mouse cells were from wild-type (C57BL/6 J; The Jackson Laboratory, Bar Harbor, ME, USA), tlr4 mutant $\left(t l r 4^{-/-}\right.$; The Jackson Laboratory, Bar Harbor, ME, USA), Lgals 9 mutant $\left(\right.$ Lgals $^{-/-}$) and transgenic (Lgals9-EGFP) JF66Gsat/Mmucd mouse pups (P1-2). Lgals $9^{-/}$mice were generated from heterozygous breeding pairs originally obtained from the Consortium of Functional Glycomics. Transgenic Lgals9:EGFP mice (Lgals9-EGFP) JF66Gsat/Mmucd strain were reconstituted from MMRRC. The experimental procedures described herein were approved by the Institutional Animal Care and Use Committee and were performed in accordance with guidelines of the National Institutes of Health.

\section{Glial cultures}

Primary microglia, astrocytes and mixed glial cultures were isolated from the forebrains of Sprague Dawley rats or mice as described previously [36,41-43]. Briefly, brains were dissected and meninges removed, and the tissue was digested with HBSS containing $0.01 \%$ trypsin and $10 \mu \mathrm{g} / \mathrm{ml}$ DNase for 15 minutes at $37^{\circ} \mathrm{C}$. The cells were then washed with DMEM containing 10\% heatinactivated FBS and 1\% penicillin-streptomycin, filtered through a sterile $70-\mu \mathrm{m}$ filter, then plated onto poly-dlysine coated $75 \mathrm{~cm}^{2}$ flasks or directly into 24-well plates for experiments using mixed glia. The medium was changed every other day and cells were treated after seven to eight days in vitro (DIV). For experiments involving mono- and co-cultures, microglia were isolated by shaking the mixed glia-containing flasks for 1 hour at $200 \mathrm{rpm}$. After removing microglia, the flasks were shaken overnight to separate oligodendrocytes from the astrocyte layer. The resulting astrocyte cultures were treated with L-leucine methyl ester $(1 \mathrm{mM})$ for 1 hour before subculturing to remove additional contaminating microglia. The purity of enriched monocultures was consistently greater than $94 \%$. Co-cultures of astrocytes and microglia were reconstituted by plating microglia directly onto enriched astrocytes. The BV2 microglial cell line (a kind gift from Dr. Monica Carson, University of California, Riverside) was cultured in complete DMEM media consisting of DMEM supplemented with 
10\% FBS (Hyclone, Logan, UT, USA), L-glutamine (1 mM), sodium pyruvate $(1 \mathrm{mM})$ (Sigma, St. Louis, MO, USA), penicillin and streptomycin (100 U each; Life Technologies, Carlsbad, CA, USA). Cells were treated with either poly (I:C) $(0$ to $50 \mu \mathrm{g} / \mathrm{ml})$ or LPS (0 to $100 \mathrm{ng} / \mathrm{ml})$ (Sigma, St. Louis, MO, USA) in DMEM medium supplemented with $0.1 \%$ BSA (Sigma, St. Louis, MO, USA) for 0 to 24 hours as indicated in the figure legend.

\section{Astrocyte dependent potentiation of microglial TNF production}

To determine the ability for astrocytes to promote microglial TNF production, we first employed a limiting dilution experiment. Here, microglia or astrocytes were plated into poly-d-lysine coated 96-well plates at a density of $5 \times 10^{4}$ cells per well. After 24 hours, increasing numbers of newly isolated microglia were added to the above established monocultures to achieve cell ratios of $0.0625: 1,0.25: 1$ and $1: 1$ as described in the Figure 1 legend. The cells were then stimulated with poly(I:C) $(50 \mu \mathrm{g} / \mathrm{ml})$ for 24 hours and TNF measured in the supernatants by ELISA.

The necessity for living astrocytes to enhance TNF production from microglia was examined by culturing microglia $\left(5 \times 10^{4}\right)$ alone or with astrocytes $\left(5 \times 10^{4}\right)$ that had either been previously fixed with ice-cold methanol for 10 minutes or incubated with PBS and washed extensively. The cultures were incubated overnight at $37^{\circ} \mathrm{C}$ and then stimulated for $0,4,8$ and 24 hours with poly(I:C). TNF production was then determined by ELISA.

To assess if the effect of astrocytes on microglia TNF production was contact-dependent, we compared the level of TNF production in microglia cultured with or without physical contact with astrocytes using methods described previously [41]. Briefly, $10 \mu$ d drops of Sylgard (polydimethylsiloxane; Dow Corning Corp., Midland, MI, USA) were spotted onto glass coverslips kept at low heat on a hot plate (Corning Inc, Corning, NY, USA) to create columns approximately $1 \mathrm{~mm}$ in height. The coverslips were sterilized, coated with poly-d-lysine, washed with sterile water and allowed to dry. Microglia were plated onto these 'bridged' coverslips, which were flipped over and placed into astrocyte cultures in 24-well plates as indicated to create non-contacting microglia/astrocytes co-cultures. For co-cultures with direct physical contacts between the two cell types, microglia were seeded directly into the astrocyte cultures. The cells were stimulated with poly(I:C) as described above for 24 hours.

\section{Immunocytochemistry}

Cells were grown on poly-d-lysine coated glass coverslips in 24-well plates as described above. Following stimulation, the cells were fixed with $4 \%$ paraformaldehyde for

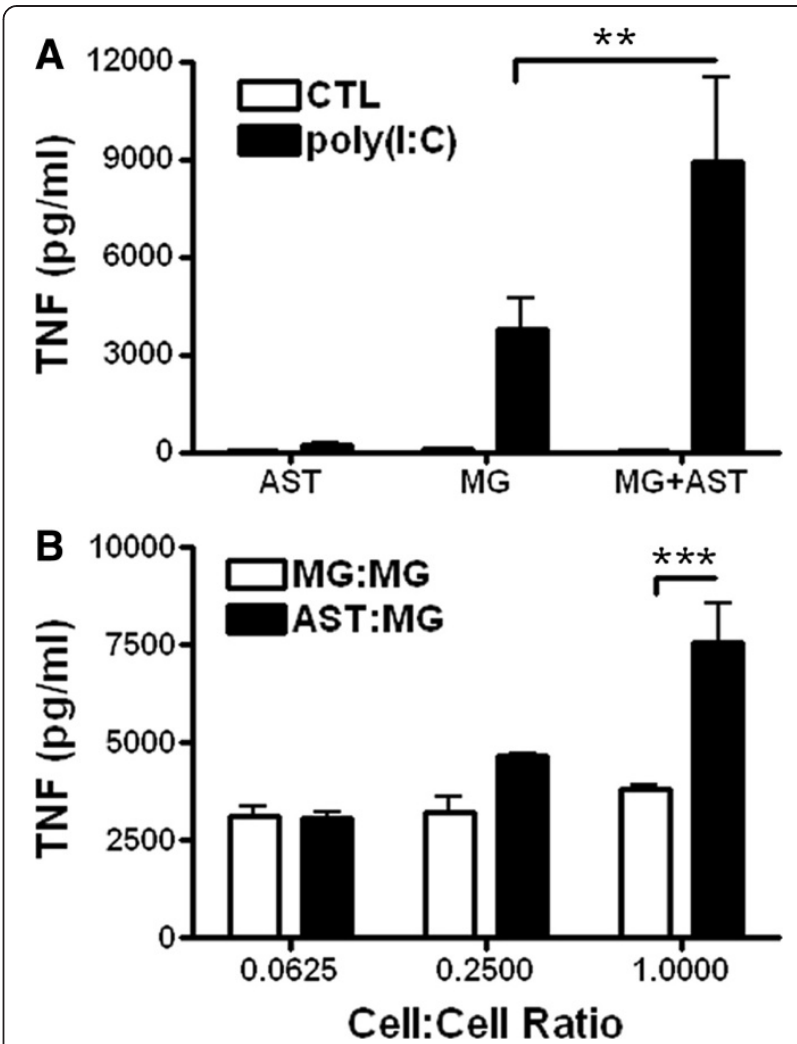

Figure 1 Astrocytes enhance microglial TNF production after poly(I:C) stimulation. (A) TNF levels from supernatants of mono- and co-cultures of microglia and astrocytes following stimulation with or without poly(l:C) $(50 \mathrm{\mu g} / \mathrm{ml})$ for 24 hours. Results are combined means \pm SE of seven independent experiments. (B) Synergistic effect of astrocytes on microglial TNF is dependent on astrocyte number Microglia were added in increasing numbers to microglia or astrocyte monocultures, and stimulated with poly(l:C) $(50 \mu \mathrm{g} / \mathrm{ml})$ for 24 hours and TNF measured from the supernatants. Results are combined means \pm SE of three independent experiments. ${ }^{*} P<0.01$, ${ }^{* *} P<0.001$.

10 minutes at room temperature then washed with PBS. The coverslips were then blocked and permeabilized with PBS containing 0.1\% Tween-20 (PBST) and 5\% goat serum for 1 hour at room temperature (RT) then incubated with chicken anti-GFP (1/400; Life Technologies, Carlsbad, CA, USA), rat anti-GFAP (1/200; Millipore, Billerica, MA, USA) or rabbit anti-Iba-1 (1/2,000; Wako, Richmond, VA, USA) overnight at $4^{\circ} \mathrm{C}$ in PBST. Subsequently, the coverslips were washed $3 \times$ with $\mathrm{PBS}$ then incubated with fluorescently labeled goat anti-chicken, anti-rat or anti-rabbit IgG (1/1,000; Life Technologies, Carlsbad, CA, USA) for 1 hour in PBST containing 5\% goat serum. After washing, the coverslips were mounted onto glass slides. Fluorescence intensity of GFP following stimulation with poly(I:C) was determined as described previously [44]. Images were captured using an Olympus DP70 digital camera mounted on an Olympus IX71 
microscope at the same exposure time (Olympus, Tokyo, Japan). The background from each image was uniformly subtracted using Photoshop then the mean fluorescence intensity of each image was determined using ImageJ $(\mathrm{NIH})$ [44]. Expression data were generated from four independent mixed glial cultures. For each time-point the mean fluorescence intensity of at least five random $10 \times$ fields were averaged per culture per time point. To determine microglial cell number in mixed cultures, Iba- $1^{+}$ cells in at least five random fields were counted using ImageJ and averaged for each mouse. Averages from each mouse were used for statistical analysis.

\section{RNA isolation and RT-qPCR}

RNA was extracted from cultured cells using Qiagen RNeasy kits according the manufacturer's instructions (Qiagen, Valencia, CA, USA). Contaminating DNA was removed by digestion with DNase I (Life Technologies, Carlsbad, CA, USA). RNA was reverse transcribed using a Promega AMV kit (Promega, Madison, WI, USA). To test for residual DNA contamination, samples that were reverse transcribed in the absence of reverse transcriptase were examined for amplification following PCR. For non-quantitative RT-PCR, 100 ng cDNA was amplified by PCR. For quantitative PCR, 10 ng of cDNA was used for amplification. The specific primer pairs were used for amplification: rat Gal-9, Forward-GGCATACCCCA CCCCAGCCT, Reverse-CAGGCAGGCTTCGCTCCTC G; rat GFAP, Forward-ACAAGGCGCTGGCAGCTGAG, Reverse-CACGTGGACCTGCTGCTGGG; rat Iba-1, ForwardCTTTTGGACTGCTGAAAGCC, Reverse-GTTTCTCCAGC ATTCGCTTC; rat Tim-3, Forward-GTCCACATTGGAGTA GGCGT, Reverse-TGAGTGCAGTCTCTGGGTTG; $\beta$-actin, Forward-AGACTTCGAGCAGGAGATGG, Reverse-CCAT CATGAAGTGTGACGTTG. For quantitative PCR, cDNA was amplified using $\mathrm{SYBR}^{\circ}$ according to the manufacturer's instructions (Life Technologies, Grand Island, NY, USA). All samples were run in duplicate or triplicate. Gene expression was normalized to $\beta$-actin and fold expression was calculated using the formula $2-{ }^{\Delta \Delta \mathrm{Ct}}$.

\section{Western blot}

The expression of galectin-9 and GFP in mixed glial cultures following poly $(\mathrm{I}: \mathrm{C})$ stimulation was determined by western blot as described previously with slight modification [36,43]. Mixed glia were stimulated with poly(I:C) ( $50 \mu \mathrm{g} / \mathrm{ml}$; Sigma, St. Louis, MO, USA) for 24 hours, lysed with Laemmli sample buffer, sonicated and frozen at $-80^{\circ} \mathrm{C}$ until use. The lysates were separated by SDS-PAGE, transferred to PVDF membranes, blocked for 1 hour at room temperature and then incubated with goat anti-mouse Gal9 (1/1,000; R\&D Systems, Minneapolis, MN, USA), chicken anti-GFP (1/1,000; Life Technologies, Carlsbad, CA, USA) or mouse anti- $\beta$-actin $(1 / 8,000$; Sigma, St. Louis, MO,
USA) overnight at $4^{\circ} \mathrm{C}$. After washing, the membranes were incubated with horseradish peroxidase conjugated anti-goat IgG (1/2,500; Jackson ImmunoResearch, West Grove, PA, USA), anti-chicken IgY (1/5,000; Thermo Scientific, Logan, UT, USA) or anti-mouse IgG (1/30,000; Jackson ImmunoResearch, West Grove, PA, USA) for 1 hour at RT. Western blot were developed with ImmunStar WesternC reagent (Bio-Rad; Hercules, CA, USA) and images were acquired with a Bio-Rad Chemidoc XRS gel documentation system (Bio-Rad; Hercules, CA, USA) and quantified with Quantity One software (Bio-Rad; Hercules, CA, USA).

\section{Cytokine measurement from culture supernatants}

Rat and mouse TNF was measured by ELISA according to the manufacturer's instructions (eBioscience; San Diego, CA, USA). Mouse microglial TNF, IL-6, IL-1 $\beta$, CCL2, IL12(p40) and IL-10 secretion following recombinant mouse galectin-9 (2 $\mu \mathrm{g} / \mathrm{ml}$; R\&D Systems, Minneapolis, MN, USA) and poly(I:C) stimulation were measured by Luminex according to the manufacturer's instructions (BioRad; Hercules, CA, USA).

\section{Flow cytometry}

All antibodies used for flow cytometry were from eBioscience (San Diego, CA, USA). To determine Tim-3 surface expression, primary microglia or BV2 cells $(1 \times$ $10^{5}$ ) were suspended in $100 \mu \mathrm{l}$ of flow cytometry buffer (PBS containing 2\% FBS), blocked with anti-CD16/32 (clone 93) for 10 minutes on ice, stained with antiCD11b-APC (clone M1/70), and anti-Tim-3-PE (clone RMT3-23) or an isotype control (IgG2a) for 20 minutes. To determine if exogenous galectin-9 could enhance microglial expression of MHC II and CD86 following poly(I:C) stimulation, cells $\left(2 \times 10^{5}\right)$ were plated onto sterile $35-\mathrm{mm}$ petri-dishes (Falcon; Becton Dickinson, Franklin Lakes, NJ, USA) and treated with recombinant galectin-9 $(2 \mu \mathrm{g} / \mathrm{ml})$ poly(I:C) $(50 \mu \mathrm{g} / \mathrm{ml})$, or both for 24 hours. The cells were detached with ice-cold Hanks' Balanced Salt Solution (HBSS) containing 5 mM EDTA, resuspended in flow cytometry buffer, blocked as described above then stained with anti-MHC II-PE (I-A/I$\mathrm{E}$; clone M5/114.15.2) or anti-CD86-PE (clone GL1) for 20 minutes on ice. Cells were gated on CD11b and the surface expression of Tim-3, MHC II or CD86 was determined by calculating mean fluorescence intensity using $\mathrm{C} 6$ Accuri system software (BD Bioscience, San Jose, CA, USA).

\section{Tim-3 inhibition}

The contribution of Tim-3 in transducing the effects of Gal-9 was determined using mixed glial cultures stimulated with or without poly(I:C) in the presence of increasing concentrations of either a neutralizing antibody to Tim-3 (clone $8 \mathrm{~B} .2 \mathrm{C} 12$ ) or isotype control 
antibody. To neutralize galectin-9/Tim-3 signaling by competitive ligation, mixed glial cultures were stimulated with or without poly(I:C) in the presence of increasing concentrations of soluble recombinant Tim-3 (Tim-3-Fc fusion protein). Levels of TNF in the supernatant were then determined by ELISA. All reagents were obtained from eBioscience (San Diego, CA, USA).

\section{Statistical analysis}

All data are presented as means \pm SE. To determine differences between experimental groups we employed twotailed Student's $t$-tests for comparisons between two groups or analysis of variance (ANOVA) followed by Bonferroni's post-hoc test to compare multiple groups. All statistics were calculated using GraphPad Prism 4 (GraphPad Software, San Diego, CA, USA). In all cases differences were considered significant when $P<0.05$.

\section{Results}

\section{Astrocytes promote TNF production from microglia}

Previously we found that microglia but not astrocytes secreted TNF following in vitro stimulation of mono- and mixed glial cultures with either LPS or poly(I:C) [41,43]. Interestingly however, while astrocyte mono-cultures failed to secrete TNF after either poly(I:C) or LPS stimulation, they drastically enhanced microglial TNF production when co-cultured with microglia (Figure 1A; Additional file 1: Figure S1A). This synergistic increase in cytokine production was dependent on the number of astrocytes, but not microglia in the co-culture as increasing the number of astrocytes cultured with microglia recapitulated the effect while increasing the number of microglia cultured with microglia failed to promote additional TNF production past a certain threshold (Figure 1B). The effect of astrocytes on microglia could be attributable to their ability to promote microglial proliferation and/or survival. However, immunocytochemical analysis as well as the measurement of lactate dehydrogenase release indicated that this was not the case (Additional file 1: Figure S1B and C). To determine if live astrocytes were necessary to enhance microglial TNF production, we fixed astrocytes with methanol prior to adding microglia (Additional file 2: Figure S2A). As observed in previous experiments, control astrocytes promoted TNF production from microglia following poly(I:C) stimulation (Additional file 2: Figure S2B). In contrast, astrocyte cultures that were fixed prior to stimulation with poly(I:C) failed to promote TNF production from microglia (Additional file 2: Figure S2A and $\mathrm{B})$. Furthermore, we determined that this effect was contact dependent (Additional file 2: Figure S2C). Together, these results demonstrate that active communication between the two cell types is required for the enhanced microglial TNF production observed in the presence of astrocytes and indicate that an astrocytederived factor(s) is likely involved in the synergy.

\section{Stimulation of mixed glial cultures with the viral mimic poly(l:C) increases galectin-9}

Human and rodent astrocytes up-regulate galectin-9 following stimulation with various proinflammatory cytokines, including IL-1 $\beta$ and TNF [36,45], both of which are released from activated microglia. Furthermore, recombinant galectin-9 is reported to enhance innate immune responses of dendritic cells [34] as well as promotes dendritic cell maturation [33]. Therefore, we first examined if poly(I:C) induces galectin- 9 expression in mixed glia. Stimulation of both rat (Figure 2A) and mouse (Figure $2 \mathrm{~B}$ ) mixed glia with poly(I:C) resulted in up-regulation of Lgals9 in a time-dependent fashion. Utilizing mixed glia from galectin-9 EGFP mice (Lgals9:EGFP) that contain EGFP expression cassette after galectin-9 promoter, we found that poly $(\mathrm{I}: \mathrm{C})$ time-dependently increased galectin- 9 expression as determined by increased fluorescence intensity of GFP (Figure 2B) as well as western blot analysis (Figure 2C). Similar responses were observed following LPS stimulation (Additional file 3: Figure S3A and B). In line with our previous results [36], microglia constitutively expressed Lgals9 (Figure 2B). However, in mixed glial cultures poly(I:C) time-dependently induced EGFP expression in cells morphologically resembled astrocytes (Figure 2D), a finding that was further confirmed by immunocytochemical analysis, which revealed co-localization of $\mathrm{GFP}^{+}$cells with $\mathrm{GFAP}^{+}$astrocytes (Figure 2E). Interestingly, microglia but not astrocyte monocultures challenged with poly(I:C) or LPS induced Lgals9 expression (Figure 2F; Additional file 3: Figure S3C). As before [36], stimulation of astrocytes but not microglia monocultures with TNF induced Lgals9 expression (Figure 2F), indicating that stimulation of microglia with poly $(\mathrm{I}: \mathrm{C})$ up-regulates galectin-9 whereas microglial-derived cytokines such as TNF causes galectin-9 up-regulation in astrocytes. Indeed, conditioned media from poly(I:C) stimulated microglia, but not media containing poly(I:C), increased Lgals9 expression in astrocytes (Figure 2G). This effect was abolished if the conditioned media was boiled prior to its addition to astrocytes (Figure $2 \mathrm{H}$ ), demonstrating that the microglial factors that induce Lgals9 transcription are heat sensitive.

\section{Astrocyte-derived galectin-9 promotes microglial TNF secretion}

To determine if galectin-9 was responsible for the synergic response from astrocytes, we stimulated control $\left(\right.$ Lgals $9^{+/+}$or $L$ gals $9^{+/-}$) and Lgals $9^{-/-}$mixed glial cultures with poly $(\mathrm{I}: \mathrm{C})$ and measured TNF secretion. There 


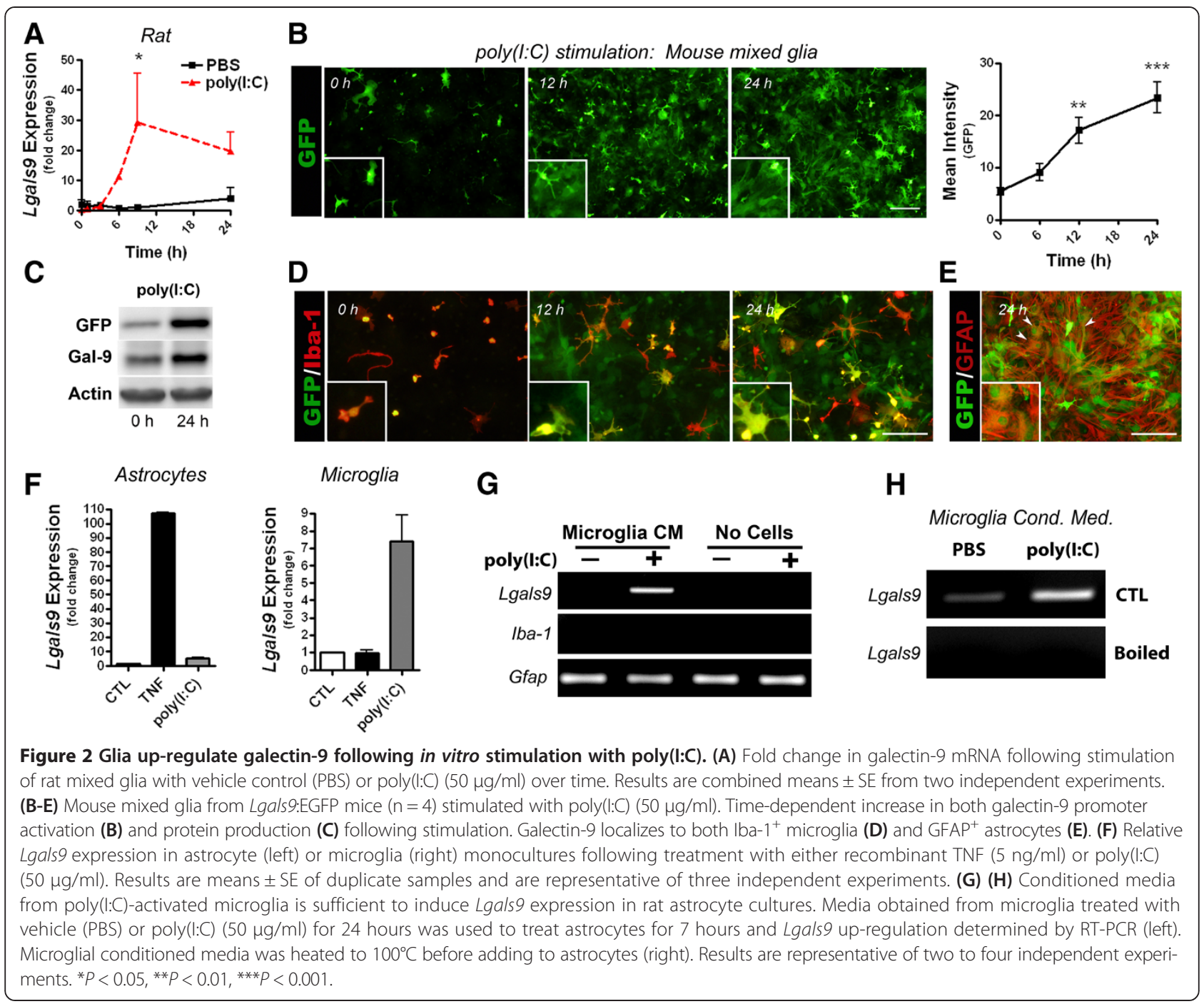

were no differences in TNF production from $\operatorname{Lgals} 9^{+/+}$ cultures compared to $\operatorname{Lgals} 9^{+/-}$cultures following poly(I: C) stimulation (not shown). However, mixed glia derived from $\operatorname{Lgals9^{-/-}}$ mice produced approximately $35 \%$ less TNF upon activation than control cultures (Figure 3A). The number of microglial cells in these cultures did not differ between genotypes (Figure 3B), thereby excluding the possibility that the decrease in TNF production was attributable to culture variation. Since microglia constitutively express galectin-9 (Figure 2D and [36]), we tested the possibility that a lack of microglial galectin-9 was responsible for the reduction in TNF. However, primary microglia isolated from $\mathrm{Lgals}_{\mathrm{S}}{ }^{+/+}$and $\operatorname{Lgals9^{-/-}}$ mice produced similar amounts of TNF after poly(I:C) stimulation (Figure 3C). Likewise, production of TNF in enriched astrocyte cultures, which is attributable to residual contaminating microglia [43] was similar between genotypes (Figure 3C). To confirm that astrocytederived galectin-9 was needed to synergize microglia
TNF production, we examined the effect of stimulation on microglia alone or co-cultured with either $L$ gals $9^{+/+}$ or Lgals $9^{-1-}$ astrocytes. Stimulation of microglia cocultured with $\operatorname{Lgals9^{+/+}}$ astrocytes produced more TNF than either microglia alone or microglia co-cultured with Lgals $^{-/-}$astrocytes, demonstrating that astrocytederived galectin- 9 promotes TNF production by microglia (Figure 3D).

\section{Recombinant galectin-9 is sufficient to promote proinflammatory responses from microglia}

Treatment of primary microglia with recombinant galectin-9, poly(I:C), or both for 24 hours changed the cell morphology (Figure 4A). To further characterize the effects of galectin-9 on the innate immune response from microglia, we examined TNF, IL-6, IL-1 $\beta$, CCL2, IL-10, and IL-12(p40) secretion after poly(I:C) stimulation. Although stimulation with exogenously added recombinant galectin-9 appeared to increase the production of most 

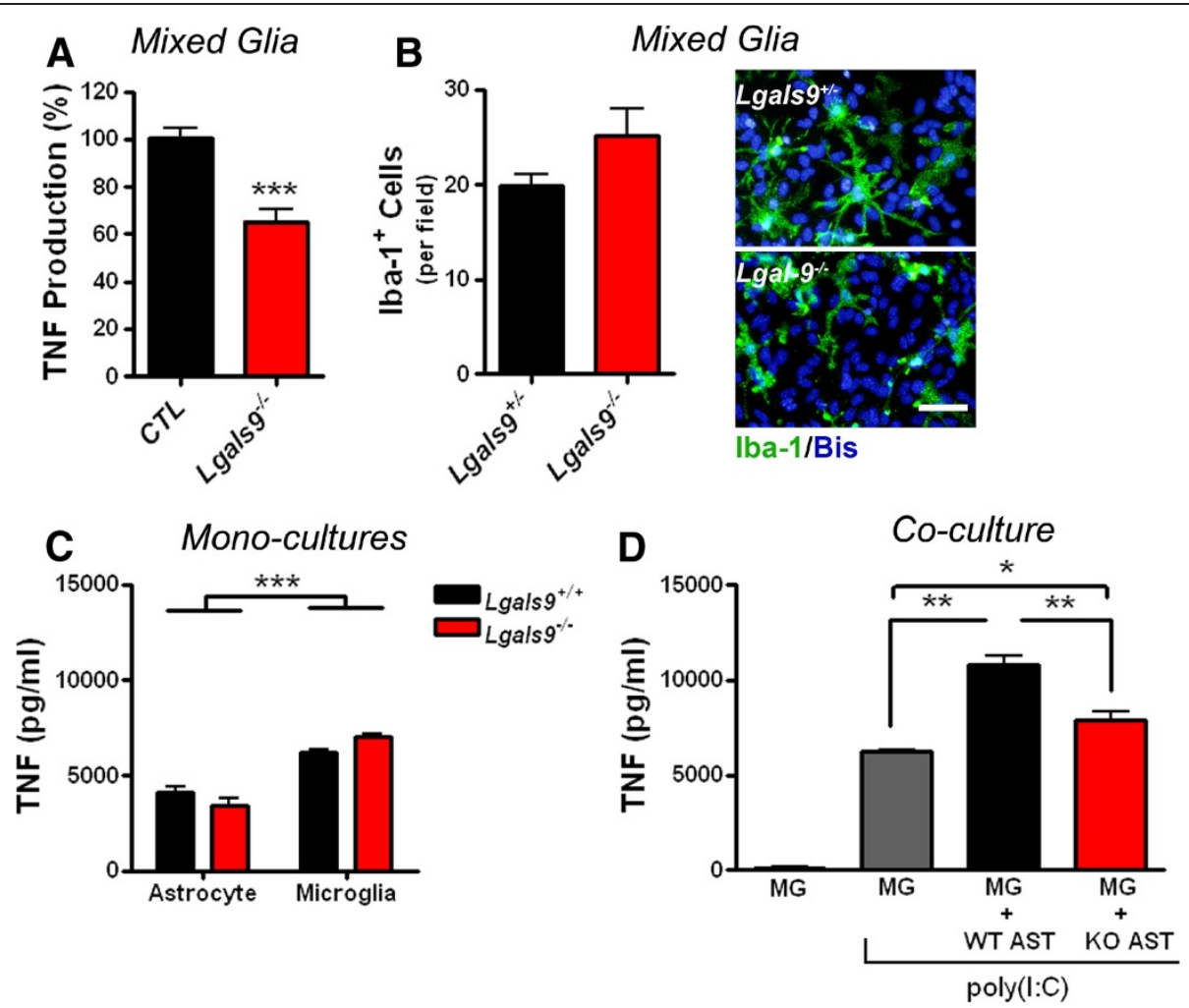

Figure 3 Astrocyte-derived galectin-9 promotes microglial TNF production. Mono-, co- or mixed glial cultures obtained from littermate control $\left(\mathrm{Lgals}_{\mathrm{s} \mathrm{9}^{+/+}}\right.$or $\left.\mathrm{Lgals9^{+/- }}\right)$ or $\mathrm{Lgals9^{-/- }}$ mice were stimulated with poly(l:C) $(25 \mathrm{\mu g} / \mathrm{ml})$ for 24 hours and TNF measured from the supernatants by ELISA. (A-B) Reduction in supernatant levels of TNF obtained from mixed glia (A) despite similar numbers of Iba- $1^{+}$microglia across genotypes

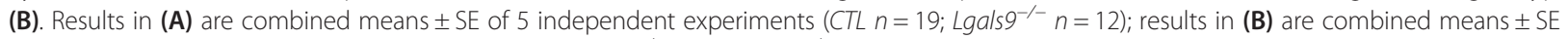

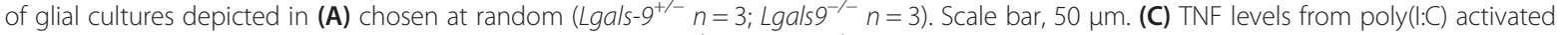
astrocyte and microglial monocultures derived from $\mathrm{Lgals}-9^{+/+}$or $\mathrm{Lgals9^{-/- }}$ mice. Results are means $\pm \mathrm{SE}$ of quadruplicate samples and are representative of two independent experiments. (D) Supernatant levels of TNF following poly(l:C) stimulation of microglial monocultures compared to co-cultures containing Lgals-9 $9^{+/+}$(wild-type (WT); black) or Lgals $9^{-/-}$(knockout (KO); red) astrocytes. Results are means \pm SE of quadruplicate samples and are representative of four independent experiments. ${ }^{*} P<0.05,{ }^{* *} P<0.01,{ }^{* *} P<0.001$.

cytokines over controls, this effect did not reach statistical significance (one-way ANOVA). In contrast, poly(I:C) stimulation increased the secretion of all measured cytokines (Figure 4B, C, D, E, F and G). Interestingly, microglial TNF was significantly increased in poly(I:C) plus galectin-9 treated cultures when compared to cultures treated with only poly(I:C) (Figure 4B). While, galectin-9 co-treatment with poly(I:C) marginally enhanced IL-6 production when compared to poly $(\mathrm{I}: \mathrm{C})$ treatment alone, the effect was not as robust as that observed for TNF (Figure 4C). Importantly, the effects of recombinant galectin-9 were unlikely to be attributable to residual endotoxin resulting from the purification of recombinant galectin- 9 as the treatment of primary microglia derived from Tlr4 null mice with galectin-9 also up-regulated TNF (Additional file 4: Figure S4).

To test if galectin-9 alters the antigen presenting capacity of microglia we cultured microglia in the presence of galectin-9, poly(I:C), or both for 24 hours and analyzed surface expression of MHC II and the co-stimulatory molecule CD86 on $\mathrm{CD} 11 \mathrm{~b}^{+}$gated cells by flow cytometry (Figure 4H-J). While poly(I:C) up-regulated the surface expression of CD86 (Figure 4I) on microglia, recombinant galectin-9 did not synergize this response. Collectively, these data demonstrate the ability of exogenous galectin- 9 to synergize poly(I:C) mediated TNF and to a lesser extent IL-6 production in microglia.

\section{Microglial Tim-3 expression does not account for the effects of galectin-9}

Galectin- 9 has been shown to increase TNF production from dendritic cells after LPS stimulation in a manner dependent on Tim-3 [34]. As such, we questioned if the effects of galectin-9 on microglia TNF production in mixed glial cultures (Figure 3A) were mediated by Tim3 signaling. To begin, we first examined if Tim-3 was expressed on microglia. Rat microglia but not astrocytes constitutively expressed Havcr2 (the gene encoding Tim-3) mRNA (Figure 5A). Similarly, flow cytometry analysis of primary mouse microglia revealed low surface 


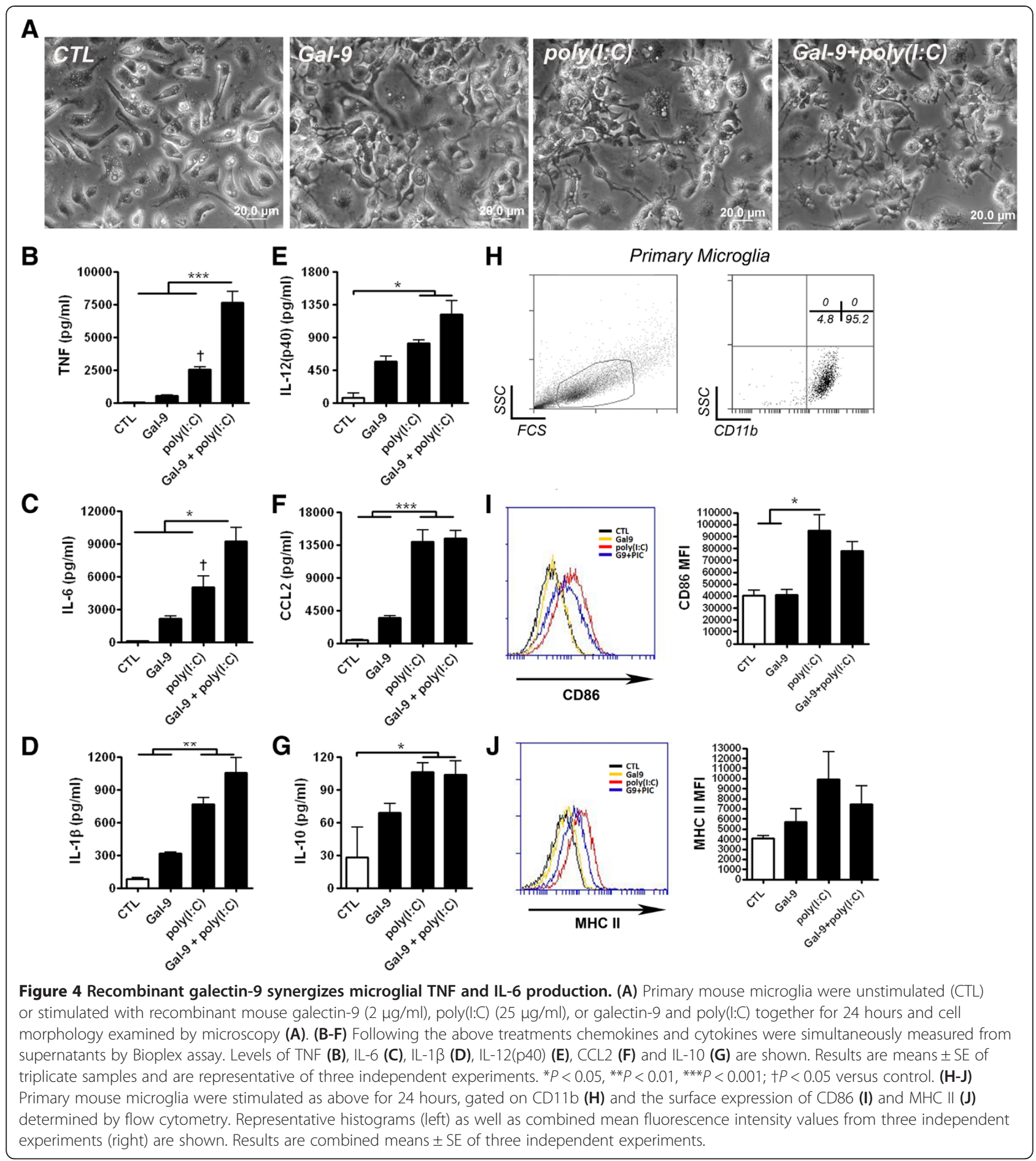

expression levels of Tim-3, whereas the mouse microglia cell line (BV2) exhibited much higher level of surface Tim-3 (Figure 5B and C). Consistent with the low Tim-3 surface expression on microglia, poly(I:C) stimulation of mixed glia in the presence of increasing concentrations of a neutralizing antibody to Tim-3 (clone 8B.2C12) did not suppress the amount of TNF produced when compared to cultures co-treated with vehicle (PBS) or an isotype control antibody (Figure 5D). Furthermore, addition of excess soluble Tim-3 (Tim-3-Fc fusion protein) also failed to alter poly(I:C)-induced TNF secretion, indicating that galectin-9 is not sequestered by Tim-3-Fc or has a higher affinity/avidity for a different glycoconjugate through which it mediates its effects (Figure 5E). 


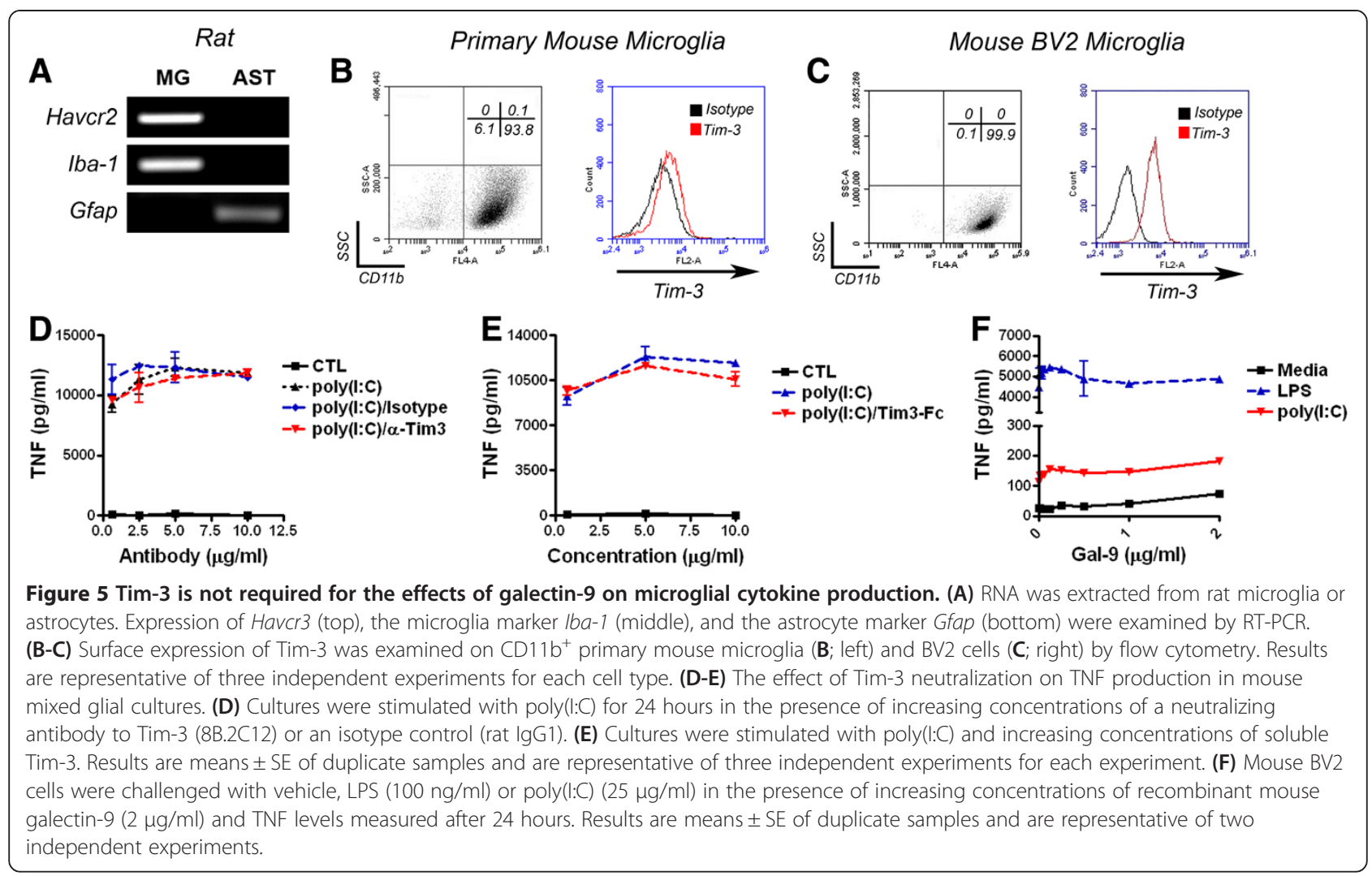

Finally, despite that BV2 cells abundantly express surface Tim-3, stimulation of this BV2 microglial cell line with recombinant galectin-9 did not increase TNF production when concurrently administered with either poly(I:C) or LPS (Figure 5F). Together these data strongly suggest that galectin-9 is capable of acting in a Tim-3 independent fashion to promote TNF production from activated microglia.

\section{Discussion}

In the current study we demonstrate that astrocytes enhance TNF production from activated microglia and that this effect is primarily dependent on galectin-9. We show that stimulation of glial cultures with the viral mimic poly(I:C) or the Gram-negative bacterial product LPS results in galectin-9 up-regulation in both microglia and astrocytes via different signaling pathways. In line with our finding that galectin-9 promotes microglial responses, we demonstrate that recombinant galectin-9 synergizes with poly(I:C) to increase TNF and IL-6 secretion from cultured microglia. Finally, our data suggest that the synergistic action of galectin- 9 and poly(I:C) on microglial TNF production is Tim-3 independent and thus is likely to be transduced through an additional, yet to be identified, glycoconjugate.

While galectin-9 is minimally expressed in the unchallenged brain, its expression has been reported to increase following acute infection with herpes simplex virus [46] and during pneumococcal meningitis [37] indicating that CNS infection likely promotes galectin-9 up-regulation. Moreover, our current and previous data provide some evidence regarding the mechanism of up-regulation for each distinct cell type within the CNS following infection. Similar to that reported for human umbilical vein endothelial cells [47], we found galectin-9 also is constitutively expressed in cerebrovascular endothelial cells (Steelman and $\mathrm{Li}$ personal observations). Additionally, we found galectin-9 expression localized to quiescent resident microglia in naïve animals (Steelman and Li personal observations), a finding that complements in vitro data from previous experiments [36]. As in HUVEC [48,49] and mesenchymal stromal cells [50], we report here that galectin-9 expression can be induced in microglia following poly(I:C) stimulation. However, despite astrocytes possessing poly(I: C) receptors and being capable of producing CCL2/5 in response to poly(I:C) stimulation [43], they themselves did not directly up-regulate galectin-9 (Figure $2 \mathrm{~F}$ ) or produce TNF [43] upon poly(I:C) stimulation. Moreover, astrocytes neither bind to LPS nor express TLR4 $[41,51,52]$ and did not increase Lgals9 expression following LPS stimulation (Additional file 3: Figure S3C). Together these findings may suggest that epigenetic regulation of inflammatory genes differ between microglia and astrocytes, a hypothesis that has great implications but has yet to be thoroughly tested. 
Nevertheless, it is likely that increased galectin-9 expression in astrocytes is dependent on proinflammatory cytokines from the surrounding milieu. The sufficiency of conditioned media from poly(I:C) activated microglia to upregulate astroglial galectin-9 expression supports this hypothesis. As such, following acute viral infection microglia and endothelial cells are likely to increase galectin-9 directly in response to pattern recognition receptor activation whereas up-regulation in astrocytes depends on proinflammatory cytokines present in the surrounding tissue.

Understanding the physiological consequence of increased galectin-9 expression within the CNS during encephalitis brought on by central or peripheral infection or autoimmunity is an important step towards understanding its therapeutic potential or pitfalls under various causes of chronic neuroinflammation. At present most previous studies have addressed the effects of galectin-9 up-regulation with regard to its function on adaptive immunity. Fewer studies have addressed the effects of galectin-9 on innate immunity with some conflicting results. For instance, it has been reported that galectin-9 can promote innate immune responses from antigen presenting cells [34] and that galectin-9 can promote dendritic cell maturation from monocyte-derived macrophages [53]. Moreover, activation of Tim-3 by galectin- 9 is reported to aid in the clearance of mycobacterial tuberculosis which is mediated by increased IL$1 \beta$ release from monocytes $[54,55]$. However, siRNA mediated abolition of Tim-3 in monocytes has been shown to cause increased production of IL-12(p70), an effect that was attributable to the cis association of galectin- 9 with Tim-3 [56,57].

To our knowledge, the current study represents the first to demonstrate a role for galectin- 9 in glial activation. Recently, Lee and Goverman have investigated the role of Tim-3 in antigen presenting cell function as it relates to EAE [58]. In these experiments they demonstrated that microglia isolated from both the quiescent and inflamed CNS express Tim-3. However, microglial Tim-3 did not alter the pathogenesis of EAE as bone marrow chimeric mice generated from Tim-3 null mice reconstituted with wild-type bone marrow did not differ in disease severity when compared to controls [58]. Furthermore, they did not find evidence to support a role for Tim-3 in modulating proinflammatory cytokine release or antigen presenting cell function from isolated dendritic cells [58]. In line with these data, the results from the current study suggest that galectin-9 can promote inflammatory cytokine production, but does so in a manner that is not, to say the least, entirely dependent on Tim-3 signaling. Some of these seemingly conflicting results may be partially explained by the promiscuous nature of galectinglycoprotein interactions. As galectins have an affinity for specific glycan moieties on glycoconjugates they would be expected to have multiple binding partners. Indeed, in addition to Tim-3 [59] galectin-9 has also been shown to bind CD40 [60], CD44 [61,62], protein disulfide isomerase [62], ICAM3, PECAM1, Integrin $\beta 1$, Integrin $\beta 2$, Integrin $\alpha \mathrm{L}$, CD2, CD6, CD26, CD45, CD48, CD107a, and CD148 [61]. Finally, the glycosylation status of proteins greatly influences galectin avidity [30,63]. Therefore, variation experimental culture conditions could potentially account for some discrepancies.

\section{Conclusion}

We have demonstrated that astrocyte-derived galectin-9 potentiates TNF and to a lesser extent IL- 6 production from microglia in vitro in a Tim-3 independent manner. Galectin-9 expression is increased in microglia following TLR ligation. However, its expression in astrocytes is likely dependent on microglia-derived cytokine production, at least in vitro. Initially, this physiological response may function to promote pathogen clearance from the CNS. However, the pathophysiological outcome of galectin-9 up-regulation within the CNS is incompletely understood. Whether glia-derived galectin-9 promotes or suppresses neuroinflammatory diseases is currently the subject of ongoing investigation.

\section{Additional files}

Additional file 1: Figure S1. Enhanced TNF production in microglia/ astrocyte co-cultures is observed following LPS stimulation. (A) TNF levels from supernatants of mono- and co-cultures of astrocytes and microglia following stimulation with or without LPS $(100 \mathrm{ng} / \mathrm{ml})$ for 24 hours. Results are combined means \pm SE of three independent experiments. (B) The number of microglia in each condition was determined by counting Iba- $1^{+}$cells in cultured treated with or without poly(l:C) $(50 \mu \mathrm{g} / \mathrm{ml})$ for 24 hours. The results are mean \pm SE of 5 to $720 x$ fields from a single experiment and are representative of 2 independent experiments. ${ }^{*} P<0.01,{ }^{* *} P<0.001$. (C) Lactate dehydrogenase (LDH) levels in culture supernatants from experiment (B).

Additional file 2: Figure S2. Live astrocytes promote microglia TNF production in a contact-dependent manner. (A) Schematic illustration of the experimental design in (B). Astrocytes were plated at $5 \times 10^{4}$ cells per well in a 96-well plate. After 22 hours the cells were incubated with either PBS (left) or ice-cold methanol (right) for 10 minutes at RT. After washing twice with warm media, $5 \times 10^{4}$ microglia were added to each well. The following day the cells were treated with poly $(\mathrm{l}: \mathrm{C})(50 \mu \mathrm{g} / \mathrm{ml})$ for $0,4,8$ and 24 hours. (B) ELISA results showing TNF secretion from microglia monocultures, microglia co-cultured with live astrocytes, microglia co-cultured with methanol-fixed astrocytes, or astrocyte monocultures following poly $(l: C)$ stimulation. Results are means \pm SE from triplicate wells. (C) Schematic illustration of the no-contact experimental design whereby microglia and astrocytes were cultured together but physically separated. The illustration is a reproduction from a similar illustration published previously [41]. (D) TNF production from microglia cultured alone or in the presence of astrocytes with (black bars) or without (white bars) contact following poly $(\mathrm{l}: \mathrm{C})(50 \mathrm{\mu g} / \mathrm{ml})$ for 24 hours. Results are means \pm SE from triplicate measurements of two separate wells per condition and are representative of three independent experiments. ${ }^{* *} P<0.01$, ${ }^{* * *} P<0.001$.

Additional file 3: Figure S3. LPS up-regulates galectin-9. (A and B) Mixed glia from Lgals9:EGFP mice were stimulated with LPS $(100 \mathrm{ng} / \mathrm{ml})$ and time-dependent increase in galectin-9 promoter activation was 
evaluated. (B) is the mean fluorescence intensity from duplicates over time Scale bar, $100 \mu \mathrm{m}$. (C) The effect of TNF (5 ng/ml) and LPS (100 ng/ml) on Lgals9 expression in rat astrocytes (left) or microglia (right) monocultures as determined by RT-qPCR. Results are means \pm SE of triplicate samples and are representative of two independent experiments.

Additional file 4: Figure S4. The effects of galectin-9 are not attributable to residual endotoxin. Microglia were isolated from wild-type C57BL/6 mice (WT) or toll-like receptor mutant mice $\left(\mathrm{Tr}_{\mathrm{r} 4^{-/}}\right)$and plated into 96 -well plates at $5 \times 10^{4}$ cells per well. Microglia from WT (left) or T/r $4^{-1-}$ (right) mice were treated with or without recombinant galectin- $9(2 \mu \mathrm{g} / \mathrm{ml})$ for 24 hours and TNF levels determined.

\section{Abbreviations}

CNS: central nervous system; EAE: experimental autoimmune encephalomyelitis; IL-6: interleukin-6; LPS: lipopolysaccharide; Tim-3: glycoprotein T-cell immunoglobulin and mucin-domain containing protein 3; TLR: toll-like receptor; TNF: tumor necrosis factor.

\section{Competing interests}

The authors declare that they have no competing interests.

\section{Authors' contribution}

AJS and JL conceived the experiments. AJS conducted the experiments, analyzed the results and drafted the manuscript. JL contributed to experimental design, data analyses and interpretation and manuscript writing. Both authors read and approved the final manuscript.

\section{Acknowledgements}

The authors would like to thank Drs. Rodger Smith $3^{\text {rd }}$, Jane Welsh, Gus Cothran, Beiyan Zhou, and Robert Chapkin for allowing the use of their equipment. The authors are grateful to Dr. Monica Carson for the BV2 cell line and Dr. Sunja Kim for the t/r $4^{-/-}$mice. This research was funded in part by $\mathrm{NIH}$ grant R01NS060017 (JL), the National Multiple Sclerosis Society research grants RG3975 and RG4586 (JL) and Postdoctoral Fellowship FG 1937 (AJS) and a Texas A\&M University Research Training Grant (AJS).

Received: 20 February 2014 Accepted: 31 July 2014

Published: 27 August 2014

\section{References}

1. Bsibsi M, Ravid R, Gveric D, van Noort JM: Broad expression of Toll-like receptors in the human central nervous system. J Neuropathol Exp Neurol 2002, 61:1013-1021.

2. Furr SR, Chauhan VS, Sterka D Jr, Grdzelishvili V, Marriott I: Characterization of retinoic acid-inducible gene-l expression in primary murine glia following exposure to vesicular stomatitis virus. J Neurovirol 2008, 14:503-513.

3. De Miranda J, Yaddanapudi K, Hornig M, Lipkin WI: Astrocytes recognize intracellular polyinosinic-polycytidylic acid via MDA-5. FASEB J 2009, 23:1064-1071

4. Farina C, Aloisi F, Meinl E: Astrocytes are active players in cerebral innate immunity. Trends Immunol 2007, 28:138-145.

5. Jack CS, Arbour N, Manusow J, Montgrain V, Blain M, McCrea E, Shapiro A, Antel JP: TLR signaling tailors innate immune responses in human microglia and astrocytes. J Immunol 2005, 175:4320-4330.

6. Engelhardt B, Ransohoff RM: The ins and outs of T-lymphocyte trafficking to the CNS: anatomical sites and molecular mechanisms. Trends Immunol 2005, 26:485-495.

7. Ransohoff RM, Cardona AE: The myeloid cells of the central nervous system parenchyma. Nature 2010, 468:253-262.

8. Kigerl KA, Gensel JC, Ankeny DP, Alexander JK, Donnelly DJ, Popovich PG: Identification of two distinct macrophage subsets with divergent effects causing either neurotoxicity or regeneration in the injured mouse spinal cord. J Neurosci 2009, 29:13435-13444.

9. Miron VE, Boyd A, Zhao JW, Yuen TJ, Ruckh JM, Shadrach JL, van Wijngaarden P, Wagers AJ, Williams A, Franklin RJ, french-Constant C: M2 microglia and macrophages drive oligodendrocyte differentiation during CNS remyelination. Nat Neurosci 2013, 16:1211-1218.

10. Williams A, Piaton G, Lubetzki C: Astrocytes - friends or foes in multiple sclerosis? Glia 2007, 55:1300-1312.
11. Taylor RA, Sansing LH: Microglial responses after ischemic stroke and intracerebral hemorrhage. Clin Dev Immunol 2013, 2013:746068.

12. Vezzani A, Aronica E, Mazarati A, Pittman QJ: Epilepsy and brain inflammation. Exp Neurol 2013, 244:11-21.

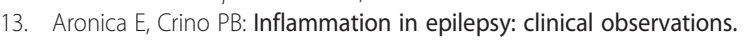
Epilepsia 2011, 52(Suppl 3):26-32.

14. Aronica E, Ravizza T, Zurolo E, Vezzani A: Astrocyte immune responses in epilepsy. Glia 2012, 60:1258-1268.

15. Glass CK, Saijo K, Winner B, Marchetto MC, Gage FH: Mechanisms underlying inflammation in neurodegeneration. Cell 2010, 140:918-934.

16. McCusker RH, Kelley KW: Immune-neural connections: how the immune system's response to infectious agents influences behavior. J Exp Biol 2013, 216:84-98

17. Dantzer R, O'Connor JC, Freund GG, Johnson RW, Kelley KW: From inflammation to sickness and depression: when the immune system subjugates the brain. Nat Rev Neurosci 2008, 9:46-56.

18. Ransohoff RM, Perry VH: Microglial physiology: unique stimuli, specialized responses. Annu Rev Immunol 2009, 27:119-145.

19. Heppner FL, Greter M, Marino D, Falsig J, Raivich G, Hovelmeyer N, Waisman A, Rulicke T, Prinz M, Priller J, Becher B, Aguzzi A: Experimental autoimmune encephalomyelitis repressed by microglial paralysis. Nat Med 2005, 11:146-152.

20. Goldmann T, Wieghofer P, Muller PF, Wolf Y, Varol D, Yona S, Brendecke SM, Kierdorf K, Staszewski O, Datta M, Luedde T, Heikenwalder M, Jung S, Prinz M: A new type of microglia gene targeting shows TAK1 to be pivotal in CNS autoimmune inflammation. Nat Neurosci 2013, 16:1618-1626.

21. Cusick MF, Libbey JE, Patel DC, Doty DJ, Fujinami RS: Infiltrating macrophages are key to the development of seizures following virus infection. J Virol 2013, 87:1849-1860.

22. Howe CL, Lafrance-Corey RG, Sundsbak RS, Sauer BM, Lafrance SJ, Buenz EJ, Schmalstieg WF: Hippocampal protection in mice with an attenuated inflammatory monocyte response to acute CNS picornavirus infection. Sci Rep 2012, 2:545.

23. Kirkman NJ, Libbey JE, Wilcox KS, White HS, Fujinami RS: Innate but not adaptive immune responses contribute to behavioral seizures following viral infection. Epilepsia 2010, 51:454-464.

24. Libbey JE, Kennett NJ, Wilcox KS, White HS, Fujinami RS: Interleukin-6, produced by resident cells of the central nervous system and infiltrating cells, contributes to the development of seizures following viral infection. J Virol 2011, 85:6913-6922.

25. Libbey JE, Kirkman NJ, Smith MC, Tanaka T, Wilcox KS, White HS, Fujinami RS: Seizures following picornavirus infection. Epilepsia 2008, 49:1066-1074.

26. Fu X, Zunich SM, O'Connor JC, Kavelaars A, Dantzer R, Kelley KW: Central administration of lipopolysaccharide induces depressive-like behavior in vivo and activates brain indoleamine 2,3 dioxygenase in murine organotypic hippocampal slice cultures. J Neuroinflammation 2010, 7:43.

27. Lawson MA, Parrott JM, McCusker RH, Dantzer R, Kelley KW, O'Connor JC Intracerebroventricular administration of lipopolysaccharide induces indoleamine-2,3-dioxygenase-dependent depression-like behaviors. I Neuroinflammation 2013, 10:87.

28. Park SE, Lawson M, Dantzer R, Kelley KW, McCusker RH: Insulin-like growth factor-I peptides act centrally to decrease depression-like behavior of mice treated intraperitoneally with lipopolysaccharide. J Neuroinflammation 2011, 8:179.

29. Liu FT, Rabinovich GA: Galectins: regulators of acute and chronic inflammation. Ann N Y Acad Sci 2010, 1183:158-182.

30. Rabinovich GA, Toscano MA: Turning 'sweet' on immunity: galectin-glycan interactions in immune tolerance and inflammation. Nat Rev Immunol 2009, 9:338-352.

31. Sato S, St-Pierre C, Bhaumik P, Nieminen J: Galectins in innate immunity: dual functions of host soluble beta-galactoside-binding lectins as damage-associated molecular patterns (DAMPs) and as receptors for pathogen-associated molecular patterns (PAMPs). Immunol Rev 2009, 230:172-187.

32. Vasta GR: Roles of galectins in infection. Nat Rev Microbiol 2009, 7:424-438.

33. Dai SY, Nakagawa R, Itoh A, Murakami H, Kashio Y, Abe H, Katoh S, Kontani K, Kihara M, Zhang SL, Hata T, Nakamura T, Yamauchi A, Hirashima M: Galectin-9 induces maturation of human monocyte-derived dendritic cells. J Immuno/ 2005, 175:2974-2981.

34. Anderson AC, Anderson DE, Bregoli L, Hastings WD, Kassam N, Lei C, Chandwaskar R, Karman J, Su EW, Hirashima M, Bruce JN, Kane LP, Kuchroo 
VK, Hafler DA: Promotion of tissue inflammation by the immune receptor Tim-3 expressed on innate immune cells. Science 2007, 318:1141-1143.

35. Wada J, Kanwar YS: Identification and characterization of galectin-9, a novel beta-galactoside-binding mammalian lectin. J Biol Chem 1997, 272:6078-6086.

36. Steelman AJ, Smith R, Welsh CJ, Li J: Galectin-9 is up-regulated in Astrocytes by tumor necrosis factor and promotes encephalitogenic T-cell Apoptosis. $J$ Biol Chem 2013, 288:23776-23787.

37. Bellac CL, Coimbra RS, Simon F, Imboden H, Leib SL: Gene and protein expression of galectin-3 and galectin-9 in experimental pneumococcal meningitis. Neurobiol Dis 2007, 28:175-183.

38. Reddy PB, Sehrawat $S$, Suryawanshi A, Rajasagi NK, Mulik S, Hirashima M, Rouse BT: Influence of galectin-9/Tim-3 interaction on herpes simplex virus-1 latency. J Immunol 2011, 187:5745-5755.

39. Gupta N, Rao PV: Transcriptomic profile of host response in Japanese encephalitis virus infection. Virol J 2011, 8:92.

40. Stancic M, van Horssen J, Thijssen VL, Gabius HJ, van der Valk P, Hoekstra D, Baron W: Increased expression of distinct galectins in multiple sclerosis lesions. Neuropathol Appl Neurobiol 2011, 37:654-671.

41. Kim S, Steelman AJ, Koito H, Li J: Astrocytes promote TNF-mediated toxicity to oligodendrocyte precursors. J Neurochem 2011, 116:53-66.

42. Li J, Ramenaden ER, Peng J, Koito H, Volpe JJ, Rosenberg PA: Tumor necrosis factor alpha mediates lipopolysaccharide-induced microglial toxicity to developing oligodendrocytes when astrocytes are present. J Neurosci 2008, 28:5321-5330.

43. Steelman AJ, Li J: Poly(l:C) promotes TNFalpha/TNFR1-dependent oligodendrocyte death in mixed glial cultures. J Neuroinflammation 2011, 8:89.

44. Steelman AJ, Thompson JP, Li J: Demyelination and remyelination in anatomically distinct regions of the corpus callosum following cuprizone intoxication. Neurosci Res 2012, 72:32-42.

45. Yoshida H, Imaizumi T, Kumagai M, Kimura K, Satoh C, Hanada N, Fujimoto K, Nishi N, Tanji K, Matsumiya T, Mori F, Cui XF, Tamo W, Shibata T, Takanashi S, Okumura K, Nakamura T, Wakabayashi K, Hirashima M, Sato Y, Satoh K: Interleukin-1 beta stimulates galectin-9 expression in human astrocytes. Neuroreport 2001, 12:3755-3758.

46. Shim JA, Park S, Lee ES, Niki T, Hirashima M, Sohn S: Galectin-9 ameliorates herpes simplex virus-induced inflammation through apoptosis. Immunobiology 2012, 217:657-666.

47. Alam S, Li H, Margariti A, Martin D, Zampetaki A, Habi O, Cockerill G, Hu Y, $\mathrm{Xu} \mathrm{Q}$, Zeng L: Galectin-9 protein expression in endothelial cells is positively regulated by histone deacetylase 3 . J Biol Chem 2011, 286:44211-44217.

48. Imaizumi T, Yoshida H, Nishi N, Sashinami H, Nakamura T, Hirashima M, Ohyama C, Itoh K, Satoh K: Double-stranded RNA induces galectin-9 in vascular endothelial cells: involvement of TLR3, PI3K, and IRF3 pathway. Glycobiology 2007, 17:12C-15C.

49. Ishikawa A, Imaizumi T, Yoshida H, Nishi N, Nakamura T, Hirashima M, Satoh K: Double-stranded RNA enhances the expression of galectin-9 in vascular endothelial cells. Immunol Cell Biol 2004, 82:410-414.

50. Gieseke F, Kruchen A, Tzaribachev N, Bentzien F, Dominici M, Muller I: Proinflammatory stimuli induce galectin-9 in human mesenchymal stromal cells to suppress T-cell proliferation. Eur J Immunol 2013, 43:2741-2749.

51. Lehnardt S, Lachance C, Patrizi S, Lefebvre S, Follett PL, Jensen FE, Rosenberg PA, Volpe JJ, Vartanian T: The toll-like receptor TLR4 is necessary for lipopolysaccharide-induced oligodendrocyte injury in the CNS. J Neurosci 2002, 22:2478-2486.

52. Holm TH, Draeby D, Owens T: Microglia are required for astroglial Toll-like receptor 4 response and for optimal TLR2 and TLR3 response. Glia 2012, 60:630-638

53. Yamauchi A, Dai SY, Nakagawa R, Kashio Y, Abe H, Katoh S, Kontani K, Hirashima M: Galectin-9 induces maturation of human monocyte-derived dendritic cells. Nihon Rinsho Meneki Gakkai Kaishi 2005, 28:381-388.

54. Jayaraman P, Sada-Ovalle I, Nishimura T, Anderson AC, Kuchroo VK, Remold HG, Behar SM: IL-1 beta promotes antimicrobial immunity in macrophages by regulating TNFR signaling and caspase-3 activation. J Immunol 2013, 190:4196-4204.

55. Jayaraman P, Sada-Ovalle I, Beladi S, Anderson AC, Dardalhon V, Hotta C, Kuchroo VK, Behar SM: Tim3 binding to galectin-9 stimulates antimicrobial immunity. J Exp Med 2010, 207:2343-2354.
56. Ma CJ, Li GY, Cheng YQ, Wang JM, Ying RS, Shi L, Wu XY, Niki T, Hirashima M, Li CF, Moorman JP, Yao ZQ: Cis association of galectin-9 with Tim-3 differentially regulates IL-12/IL-23 expressions in monocytes via TLR signaling. PLOS ONE 2013, 8:e72488.

57. Wang JM, Shi L, Ma CJ, Ji XJ, Ying RS, Wu XY, Wang KS, Li G, Moorman JP, Yao ZQ: Differential regulation of interleukin-12 (IL-12)/IL-23 by Tim-3 drives $\mathrm{T}(\mathrm{H}) 17$ cell development during hepatitis $\mathrm{C}$ virus infection. J Virol 2013, 87:4372-4383.

58. Lee SY, Goverman JM: The influence of T cell Ig mucin-3 signaling on central nervous system autoimmune disease is determined by the effector function of the pathogenic T cells. J Immunol 2013, 190:4991-4999.

59. Sabatos CA, Chakravarti S, Cha E, Schubart A, Sanchez-Fueyo A, Zheng XX Coyle AJ, Strom TB, Freeman GJ, Kuchroo VK: Interaction of Tim-3 and Tim-3 ligand regulates T helper type 1 responses and induction of peripheral tolerance. Nat Immunol 2003, 4:1102-1110.

60. Vaitaitis GM, Wagner DH Jr: Galectin-9 controls CD40 signaling through a Tim-3 independent mechanism and redirects the cytokine profile of pathogenic T cells in autoimmunity. PLOS ONE 2012, 7:e38708.

61. Clayton KL, Haaland MS, Douglas-Vail MB, Mujib S, Chew GM, Ndhlovu LC, Ostrowski MA: T cell Ig and mucin domain-containing protein 3 is recruited to the immune synapse, disrupts stable synapse formation, and associates with receptor phosphatases. J Immunol 2014, 192:782-791

62. Bi S, Hong PW, Lee B, Baum LG: Galectin-9 binding to cell surface protein disulfide isomerase regulates the redox environment to enhance T-cell migration and HIV entry. Proc Natl Acad Sci U S A 2011, 108:10650-10655.

63. Grigorian A, Demetriou M: Manipulating cell surface glycoproteins by targeting N-glycan-galectin interactions. Methods Enzymol 2010, 480:245-266.

doi:10.1186/s12974-014-0144-0

Cite this article as: Steelman and Li: Astrocyte galectin-9 potentiates microglial TNF secretion. Journal of Neuroinflammation 2014 11:144.

\section{Submit your next manuscript to BioMed Central and take full advantage of:}

- Convenient online submission

- Thorough peer review

- No space constraints or color figure charges

- Immediate publication on acceptance

- Inclusion in PubMed, CAS, Scopus and Google Scholar

- Research which is freely available for redistribution

Submit your manuscript at www.biomedcentral.com/submit
C Biomed Central 